\title{
Causas del Desinterés por la Práctica Deportiva en Estudiantes de Bachillerato de la Amazonia Ecuatoriana
}

\section{Causes of Disinterest in Sports Practice in High School Students from the Ecuadorian Amazon}

\author{
Carlos Julio Bonito-Cisneros \\ carlos.bonito.95@est.ucacue.edu.ec \\ Universidad Católica de Cuenca, Azogues \\ Ecuador \\ https://orcid.org/0000-0002-1558-4550 \\ Zoila Guillermina Torres-Palchisaca \\ ztorresp@ucacue.edu.ec \\ Universidad Católica de Cuencax|, Azogues, Cuenca \\ Ecuador \\ https://orcid.org/0000-0003-3078-6465
}

Recepción: 30 de agosto 2021

Revisado: 20 de septiembre 2021

Aprobación: 15 de noviembre 2021

Publicación: 01 de diciembre 2021 
Revista Arbitrada Interdisciplinaria KOINONIA

Año VI. Vol VI. N4. Edición Especial: Educación III. 2021

Hecho el depósito de Ley: FA2016000010

ISSN: 2542-3088

FUNDACIÓN KOINONIA (F.K). Santa Ana de Coro. Venezuela.

Carlos Julio Bonito-Cisneros; Zoila Guillermina Torres-Palchisaca

\title{
RESUMEN
}

El tema "Causas del Desinterés por la Práctica Deportiva en Estudiantes de Bachillerato de la Amazonia Ecuatoriana" tiene por objeto descubrir las causas de este desinterés. Descriptiva, aplicando una encuesta on line, con una muestra de 327 estudiantes. Uno de los hallazgos más importantes, respecto a la actividad física es, que los estudiantes practican el trote y la caminata, pero otros no hacen ningún tipo de actividad deportiva, por la falta de tiempo. Conclusión, los estudiantes, no practican deporte por desconocimiento de los beneficios en su salud y de la existencia de escenarios deportivos en la localidad, como el centro de alto rendimiento, que permanece vacío. La desmotivación provoca la no adherencia a la práctica deportiva. La inadecuada distribución del tiempo libre constituye otro limitante, se justifica porque los estudiantes alegan que no tienen tiempo suficiente porque están dedicados a sus estudios.

Descriptores: Rendimiento escolar; actividad juvenil; competición. (Palabras tomadas del Tesauro UNESCO).

\begin{abstract}
The theme "Causes of Disinterest in Sports Practice in High School Students in the Ecuadorian Amazon" aims to discover the causes of this disinterest. Descriptive, applying an online survey, with a sample of 327 students. One of the most important findings regarding physical activity is that students practice jogging and walking, but others do not do any type of sports activity, due to lack of time. Conclusion, the students do not practice sports due to ignorance of the benefits to their health and the existence of sports venues in the town, such as the high performance center, which remains empty. Demotivation causes non-adherence to sports practice. The inadequate distribution of free time constitutes another limitation, it is justified because the students allege that they do not have enough time because they are dedicated to their studies.
\end{abstract}

Descriptors: Academic achievement; youth activities; competition. (Words taken from the UNESCO Thesaurus). 


\section{INTRODUCCIÓN}

La presencia de adolescentes deportistas en los escenarios deportivos de la ciudad de Macas, se ha reducido en la actualidad comparado con lo que sucedía en años anteriores, antes era muy fácil encontrar los gimnasios llenos de deportistas, que trabajaban muy duro para poder alcanzar un objetivo en común que es la de participar en Juegos Nacionales representando a la provincia y así poder romper marcas que los ayuden a clasificar a eventos de mayor envergadura.

Lastimosamente hoy en día, los escenarios aparecen vacíos, ya que muy pocos jóvenes ocupan su tiempo libre en practicar un deporte. En este sentido, la práctica de actividad física controlada y programada produce numerosos beneficios para la salud, por lo tanto; comentan que el motivo más citado de este abandono es la falta de tiempo, seguido de la preferencia por otras actividades en el tiempo libre y la pereza y desgana (Macarro et al. 2009).

Sin embargo, parece ser generalizado que durante la pubertad en la Amazonía ecuatoriana, específicamente en Morona Santiago, hay muy poco interés por el ejercicio, muchos adolescentes prefieren llevar una vida sedentaria, o en ocasiones enfocar sus energías en actividades que no tienen ningún beneficio personal. De esta forma, es fundamental que los educadores deportivos difundan que la práctica de actividad física incide positivamente en el desarrollo individual y social de la persona (Barbosa-Granados \& Urrea-Cuéllar, 2018).

De lo expuesto, resulta necesario realizar este estudio que tiene por objetivo determinar las causas por las cuales los adolescentes ya no se motivan para practicar deportes, el desinterés que hay en esta población comprendida en edades de 15 a 18 años para ocupar su tiempo libre en otras cosas que no sean la actividad física. Los resultados serán llevados hasta los organismos deportivos para que, desde estos, se pueda buscar estrategias para captar más deportistas, gestiones que ayuden a reclutar la mayor cantidad de jóvenes para que dediquen su tiempo libre al deporte y actividad física. 


\section{Referencial teórico}

\section{La práctica deportiva}

La práctica deportiva según (Giakoni et al. 2020), tiene un elemento en común que es el movimiento, originado por el cuerpo humano. Todo movimiento del cuerpo que es producido por los músculos y el esqueleto requieren un gasto energético y se realiza de manera determinada y con objetivos concretos que generan los varios deportes que el ser humano realiza, en este caso la práctica deportiva será la actividad física deportiva orientada con objetivos de salud y de rendimiento deportivo.

\section{Los adolescentes}

La adolescencia es una etapa más fascinante y compleja del ser humano, donde asume nuevas responsabilidades y un nuevo sentimiento de independencia, identidad, práctica de valores y desarrollo de habilidades que le permiten en su la juventud y la edad adulta para ser seres beneficiosos para la sociedad en la que viven. Se considera adolescencia a los 10 a 18 años de edad, esta etapa es donde cursan el bachillerato y se preparan para ingresar al ámbito empresarial o profesional ingresando a la universidad (Roach, 2018).

\section{Desinterés por el deporte.}

En la actualidad los adolescentes o estudiantes secundarios se han vuelto apáticos a la práctica deportiva, generado por varios distractores sociales, comunicativos y la lúdica virtual lo que ha producido que se alejen de las canchas deportivas y a través de celulares están jugando o practicando deportes de manera virtual. Es por ello que a continuación se abortarán temáticas de motivación que hace falta en los adolescentes para poder dar soluciones a la problemática que se está enfrentando en los establecimientos educativos no solo de la amazonia sino de todo el país.

Para contextualizar el tema de investigación es necesario hacer una breve referencia a la Teoría de la Autodeterminación, que no es más que un proceso intencional y continuo, 
cuyas raíces más profundas se desarrollan temprano en la vida. El mismo que se relaciona con la capacidad de expresar las preferencias, decisiones, autonomía y cierto tipo de control del entorno (Erwin, et al. 2009).

Por otro lado, (Moreno \& Martínez, 2006), plantean que la teoría de la autodeterminación es una macro teoría de la motivación humana, la cual tiene una relación directa con el desarrollo y funcionamiento de la personalidad dentro de la ecología social. Esta teoría analiza el grado en que los comportamientos humanos son volitivos o auto determinados, es decir, el grado en que las personas realizan sus acciones al más alto nivel de reflexión y se involucran en acciones con un sentido de elección.

De lo anterior podemos citar a (Deci \& Ryan,1985), quienes indican que "la motivación intrínseca es la fuente de energía fundamental para la naturaleza activa del organismo. Su reconocimiento destacó los puntos importantes de que no todos los comportamientos se basan en el impulso, ni son una función de controles externos" (p. 12). El organismo humano es activo porque posee una motivación interna fundamental propia de su ser, por lo que no todos los comportamientos tienen como base el impulso o atiende a controles externos. Por lo tanto, cuando las personas están intrínsecamente motivadas, actúan por su propio interés, "porque es divertido", y por la sensación de desafío que esta actividad proporciona. Este comportamiento se produce de forma espontánea y no se realiza por ningún motivo instrumental (Reeve, 2016).

Para completar la idea se puede citar a González (2008), quien señala: "La motivación intrínseca es aquella que encuentra satisfacción en la consecución de la meta que se propone". (p. 124). En cuanto a la motivación extrínseca, (Reeve, 2016), explica que la motivación extrínseca se basa en incentivos y consecuencias en el entorno, y surge de algunas causas independientes de la propia actividad y que está dentro del contrato conductual "haz esto y obtendrás aquello" por lo que se considera una razón creada por el medio ambiente. Esto nos permite establecer una diferencia fundamental entre la motivación intrínseca y extrínseca, que es la fuente que da energía y dirige el comportamiento humano. 


\section{La adherencia al ejercicio.}

En este sentido, (De-Andrade-Bastos et. Al. 2006), (Hong et al. 2008), señalan con respecto a la adherencia al ejercicio, que las personas físicamente activas son las que tienen más probabilidades de llevar a cabo conductas saludables, y para evitar conductas de riesgo para su salud, se asume que el ejercicio de actividad física hace del fenómeno de la adherencia cada vez más uno de los pilares esenciales de la salud. El término adherencia no siempre se utiliza de forma coherente. Actualmente, los cuatro componentes básicos de adherencia, presencia, finalización, duración e intensidad se utilizan indistintamente en varios estudios.

\section{MÉTODO}

Esta investigación fue descriptiva, ya que se conocieron las características de las variables transversales, pues las herramientas de evaluación se realizaron en un tiempo determinado, con un método analítico deductivo, por cuanto las posibles causas que provocan el abandono de los deportes en estudiantes de secundaria. Para el estudio se examinaron 700 estudiantes de secundaria del Colegio de Bachillerato Macas de la ciudad de Macas. En una muestra de 327 estudiantes, esto se determinó mediante un muestreo de conveniencia no verosímil.

La técnica utilizada fue la encuesta, la misma que validada por expertos del área y el instrumento fue el cuestionario con 10 preguntas. La encuesta se realizó on line en Google forms en vista de que los estudiantes se encontraban confinados por la pandemia.

Una vez que se aplicó la encuesta se procedió a tabular los datos mediante el programa Excel, esta información fue colocada en tablas estadísticas con los respectivos indicadores, frecuencias y porcentajes, finalmente se procedió a analizar e interpretar los datos estadísticos que nos ofrecieron las bases para recabar las conclusiones. 


\section{RESULTADOS}

\section{Tabla 1.}

Resumen de la encuesta aplicada a los estudiantes del Colegio de Bachillerato Macas, sobre las causas del desinterés por la práctica deportiva en Estudiantes de Bachillerato de la Amazonía Ecuatoriana.

\begin{tabular}{|c|c|c|}
\hline 1.Edad & Frecuencia & Porcentaje \\
\hline 15 & 92 & $28,13 \%$ \\
\hline 16 & 106 & $32,42 \%$ \\
\hline 17 & 107 & $32,72 \%$ \\
\hline 18 & 22 & $6,73 \%$ \\
\hline Total & 327 & $100,00 \%$ \\
\hline 2.Genero & Frecuencia & Porcentaje \\
\hline Masculino & 152 & $46,48 \%$ \\
\hline Femenino & 174 & $53,21 \%$ \\
\hline Otros & 1 & $0,31 \%$ \\
\hline Total & 327 & $100,00 \%$ \\
\hline 3. ¿Con qué frecuencia a la semana realiza prácticas deportivas? & Frecuencia & Porcentaje \\
\hline Un día & 61 & $18,65 \%$ \\
\hline Dos días & 62 & $18,96 \%$ \\
\hline Tres días & 53 & $16,21 \%$ \\
\hline Todos los días & 94 & $28,75 \%$ \\
\hline Nunca & 57 & $17,43 \%$ \\
\hline Total & 327 & $100,00 \%$ \\
\hline $\begin{array}{l}\text { 4. ¿Cuál es el tiempo que usted ocupa al día, para realizar una } \\
\text { rutina de prácticas deportivas? }\end{array}$ & Frecuencia & Porcentaje \\
\hline 1 hora & 128 & $39,14 \%$ \\
\hline 2 horas & 90 & $27,52 \%$ \\
\hline 3 horas & 36 & $11,01 \%$ \\
\hline 4 horas & 11 & $3,36 \%$ \\
\hline Ninguna & 62 & $18,96 \%$ \\
\hline Total & 327 & $100,00 \%$ \\
\hline 5. ¿Qué tipo de actividad deportiva realiza usted? & Frecuencia & Porcentaje \\
\hline Camina, trote & 104 & $31,80 \%$ \\
\hline Fútbol & 90 & $27,52 \%$ \\
\hline Baloncesto & 16 & $4,89 \%$ \\
\hline
\end{tabular}


Revista Arbitrada Interdisciplinaria KOINONIA

Año VI. Vol VI. N4. Edición Especial: Educación III. 2021

Hecho el depósito de Ley: FA2016000010 ISSN: 2542-3088

FUNDACIÓN KOINONIA (F.K). Santa Ana de Coro. Venezuela.

Carlos Julio Bonito-Cisneros; Zoila Guillermina Torres-Palchisaca

\begin{tabular}{|c|c|c|}
\hline Ciclismo & 14 & $4,28 \%$ \\
\hline Otros & 48 & $14,68 \%$ \\
\hline Ninguna & 69 & $21,10 \%$ \\
\hline Total & 327 & $100,00 \%$ \\
\hline $\begin{array}{l}6 \text { ¿Indique una razón por la cual no asistiría a las prácticas deportivas que } \\
\text { se ofrecen en la localidad? }\end{array}$ & Frecuencia & Porcentaje \\
\hline Por desconocimiento & 106 & $32,42 \%$ \\
\hline Por no tener tiempo suficiente & 114 & $34,86 \%$ \\
\hline No tener recursos económicos & 48 & $14,68 \%$ \\
\hline Tengo ocupaciones familiares & 36 & $11,01 \%$ \\
\hline No tiene el apoyo de los padres & 23 & $7,03 \%$ \\
\hline Total & 327 & $100,00 \%$ \\
\hline 7. ¿En qué ocupa su tiempo libre? & Frecuencia & Porcentaje \\
\hline Redes sociales & 113 & $34,56 \%$ \\
\hline Juegos virtuales & 56 & $17,13 \%$ \\
\hline Deportes & 115 & $35,17 \%$ \\
\hline Nada & 43 & $13,15 \%$ \\
\hline Total & 327 & $100,00 \%$ \\
\hline $\begin{array}{l}8 \text { ¿Cuál sería su preocupación más grande, si le invitaran a realizar } \\
\text { prácticas deportivas? }\end{array}$ & Frecuencia & Porcentaje \\
\hline Sufrir lesiones & 107 & $32,72 \%$ \\
\hline Bajar el rendimiento académico en el colegio & 106 & $32,42 \%$ \\
\hline Instalaciones deportivas alejadas de mi residencia & 67 & $20,49 \%$ \\
\hline Mucho esfuerzo físico & 47 & $14,37 \%$ \\
\hline Total & 327 & $100,00 \%$ \\
\hline $\begin{array}{l}\text { 9. ¿Estaría de acuerdo en que se propongan programas de apoyo para } \\
\text { deportistas? }\end{array}$ & Frecuencia & Porcentaje \\
\hline Completamente de acuerdo & 269 & $82,26 \%$ \\
\hline Medianamente de acuerdo & 53 & $16,21 \%$ \\
\hline En desacuerdo & 5 & $1,53 \%$ \\
\hline Total & 327 & $100,00 \%$ \\
\hline $\begin{array}{l}\text { 10. Si le ofrecieran apoyo para practicar algún deporte, ¿cuál de las } \\
\text { siguientes opciones serían recomendadas? }\end{array}$ & Frecuencia & orcentaje \\
\hline Económica & 175 & $53,52 \%$ \\
\hline Movilidad & 87 & $26,61 \%$ \\
\hline Alimentación & 39 & $11,93 \%$ \\
\hline Estadía & 26 & $7,95 \%$ \\
\hline Total & 327 & $100,00 \%$ \\
\hline
\end{tabular}

Fuente: Encuesta. 
Revista Arbitrada Interdisciplinaria KOINONIA

Año VI. Vol VI. N4. Edición Especial: Educación III. 2021

Hecho el depósito de Ley: FA2016000010 ISSN: $2542-3088$

FUNDACIÓN KOINONIA (F.K). Santa Ana de Coro. Venezuela.

Carlos Julio Bonito-Cisneros; Zoila Guillermina Torres-Palchisaca

\section{DISCUSIÓN}

El tamaño de la muestra fue de 327 estudiantes, del género masculino 152 que corresponde al $46,48 \%$, del género femenino 174 que corresponde al $53,21 \%$ y a otros 1 que corresponde al 0,31\%. La edad de los estudiantes encuestado fue; de 15 años 92 estudiantes que representa el 28,13\%, de 16 años, 106 estudiantes que representa el $32,42 \%$, de 17 años, 107 estudiantes que representa el 32,72\% y de 18 años, 22 estudiantes que represente el $6,73 \%$.

Con respecto a la frecuencia con la que los estudiantes realizan prácticas deportivas, se observa que existen valores bastante similares, siendo el valor más relevante de 28,75 $\%$ que lo practican todos los días, lo que es un valor bastante bajo con relación a la muestra, sin embargo, (Barbosa-Granados, S. H., \& Urrea-Cuéllar, 2018), aseveran que, si bien no hay una norma que establezca la frecuencia de realizar prácticas deportivas durante la semana, lo que recomendable es realizarlo todos los días, lo que es mucho mejor que nunca practicar.

Con respecto a la actividad deportiva que más realizan los estudiantes adolescentes son: el trote y la caminata con un porcentaje del $31,80 \%$, y se observa que el $21,10 \%$, no hace ningún tipo de actividad, este último dato se lo puede relacionar con lo dicho en la pregunta 6 , donde los adolescentes en un $34,86 \%$, indican que no practican deporte por la falta de tiempo, ante esto (Macarro et al. 2009), indican que, los adoescentes en la acutalidad priorizan su tiempo en juegos virtuales que les quita el tiempo necesario para poder realizar práctica deportiva. Lo dicho por los autores mencionados, se relaciona grandemente con los datos obtenidos en la pregunta 7 , donde el 51,69\% ocupan su tiempo libre en las redes sociales y juegos virtuales. Estos datos resultan muy preocupantes ya que si al $51,69 \%$ le sumamos el $13,15 \%$ de adolescentes que no hacen nada en su tiempo libre, se obtiene un $64,84 \%$ de adolescentes que desperdician su tiempo libre, es decir no existe una adherncia al ejercicio y la practica deportiva, lo cual se presenta por la falta de motivación y estrategias metodológicas adecuadas que muchas veces parte desde la clase de Educación Física. 
Al cuestionar a los adolecentes encuestado sobre cual es el temor mas grande que le impide realizar prcticas deportivas, el $32,72 \%$ es sufrir algun tipo de lesión, situación que es confirmada por (Reeve, 2016), al mencionar que los riesgos en el deporte son variados, sin embargo los adolescentes siempre tien algún temor para realizarlo, siendo estos los riesgos de lesiones físicos en caídas y golpes en alguna parte del cuerpo.

Por otro lado, (Navarro-Aburto et al. 2017), indican que se principal temor esta dirigido a la baja en el rendimiento academico, los adolescentes siempre tienen temores $u$ obstáculos que no les permiten realizar actividades de su preferencias como la práctica de deportes o jobbies, entre estos temores están las preocupaciones de su accionar pedagógica que predicen que si se dedican al deporte ban a obtener bajo rendimiento. Sin embargo es preciso que se pueda administar de la mejor manera el tiempo libre del que disponen la mayoria de adolescentes, todo esto con la colabroación y orientación de padres de familia, entrenadores y las instituciones educativas, estas ultimas sobre todo con la dosificación adecuada de tareas.

Definitivamente para que el deporte surja es necesario el apoyo de los organismos gubernamantales, que inviertan en los jóvenes deportistas, muchos de ellos con un enorme talento, que en la mayoría de los casos se pierde por esta falta de interés, es así que en la encuesta realizada ,el $82,26 \%$ de estudiantes.están de acuerdo que se realice un programa de apoyo para deportistas de la localidad amazónica. Dentro de estos programas de apoyo los estuiantes en un 53\% manifiestan que estarían dispuestos a recibir apoyo económico para practicar algún deporte, considerando que éste, no necesariamente es dinero, sino se refiere a trasporte, alimentación, implementación deportiva, entrenadores, etc. puesto que como manifiesta (Britapaz-Avarez \& Del-ValleDíaz, 2015), los adolescentes tienen inconvenientes para acceder a los campos deportivos por situaciones de tipo economía. 


\section{CONCLUSIONES}

Una vez concluido el proceso de investigación se pudo determinar las causas del desinterés para ejercicios deportivos con estudiantes de secundaria en la Amazonía ecuatoriana, siendo estas: por desconocimiento de los beneficios de la práctica deportiva cotidiana en su salud y además y de los distintos escenarios deportivos con los que se cuenta en la localidad como son: el centro de alto rendimiento, donde se puede practicar judo, pesas, karate, futbol, natación, tenis de mesa, etc. en polideportivo que se puede practicar box y gimnasia, y en el coliseo 29 de mayo se practica baloncesto, volibol y ajedrez, escenarios que prácticamente permanecen vacíos.

Por otro lado, la escasa distribución del tiempo libre constituye una gran limitación para la práctica de deportes, sin embargo, esto se justifica por el hecho de que los estudiantes manifiestan no disponer del tiempo suficiente para dedicarse al estudio. $\mathrm{Y}$ el miedo a bajar el rendimiento escolar siempre está latente. La desmotivación que surge primero de las propias lecciones de educación física, debido a la falta de metodologías que promuevan la adherencia a la práctica deportiva. Otro miedo que tienen los adolescentes es el de lesionarse, los dolores provocados por las lesiones producidas durante el deporte y el entrenamiento son comunes, por lo que muchos de ellos sienten el miedo a practicar deporte.

Finalmente, los estudiantes no practican ningún deporte por que no cuentan con los recursos económicos suficientes, las consecuencias de la pandemia han reducido el poder adquisitivo de las familias de la amazonia, lo que provocado que los estudiantes no tengan la opción de practicar algún deporte en la actualidad.

\section{FINANCIAMIENTO}

No monetario. 
Revista Arbitrada Interdisciplinaria KOINONIA

Año VI. Vol VI. N4. Edición Especial: Educación III. 2021

Hecho el depósito de Ley: FA2016000010 ISSN: 2542-3088

FUNDACIÓN KOINONIA (F.K). Santa Ana de Coro. Venezuela.

Carlos Julio Bonito-Cisneros; Zoila Guillermina Torres-Palchisaca

\section{AGRADECIMIENTO}

A la Universidad Católica de Cuenca; por todo el apoyo brindado en la motivación y desarrollo de esta investigación.

\section{REFERENCIAS CONSULTADAS}

Barbosa-Granados, S. H., \& Urrea-Cuéllar, Á. (2018). Influencia del deporte y la actividad física en el estado de salud físico y mental: una revisión bibliográfica [Influence of sport and physical activity on physical and mental health status: a literature review]. Katharsis, (25), 155-173. https://doi.org/10.25057/25005731.1023

Britapaz-Avarez, L, \& Del-Valle-Díaz, J. (2015). Significado del deporte en la dimensión social de la salud [Significance of sport in the social dimension of health]. Salus, 19,28-33. https://www.redalyc.org/pdf/3759/375943551006.pdf

de-Andrade-Bastos, A., Salguero, A., González-Boto, R., \& Marquez, S. (2006). Motives for participation in physical activity by Brazilian adults. Perceptual and motor skills, 102(2), 358-367. https://doi.org/10.2466/pms.102.2.358-36

Deci, E., \& Ryan, R. (1985). Intrinsic Motivation and Self Determination in Human Behavior. New York: A Division Of Plenum Publishing Corporation. https://link.springer.com/book/10.1007/978-1-4899-2271-7

Erwin, E. J., Brotherson, M. J., Palmer, S. B., Cook, C. C., Weigel, C. J., \& Summers, J. A. (2009). How to Promote Self-Determination for Young Children With Disabilities: Evidenced-Based Strategies for Early Childhood Practitioners and Families. Young Exceptional Children, 12(2), 27-37. https://doi.org/10.1177/1096250608329611

Giakoni, F., Paredes Bettancourt, P., \& Duclos-Bastías, D. (2021). Educación Física en Chile: tiempo de dedicación y su influencia en la condición física, composición corporal y nivel de actividad física en escolares [Physical Education in Chile: time spent and its influence on physical condition, body composition, and I). Retos, 39, 24-29. https://doi.org/10.47197/retos.v0i39.77781

González, D. (2008). Psicología de la motivación [psychology of motivation]. Habana: Editorial Ciencias Médicas. 
Revista Arbitrada Interdisciplinaria KOINONIA

Año VI. Vol VI. N4. Edición Especial: Educación III. 2021

Hecho el depósito de Ley: FA2016000010 ISSN: 2542-3088

FUNDACIÓN KOINONIA (F.K). Santa Ana de Coro. Venezuela.

Carlos Julio Bonito-Cisneros; Zoila Guillermina Torres-Palchisaca

Hong, S. Y., Hughes, S., \& Prohaska, T. (2008). Factors affecting exercise attendance and completion in sedentary older adults: a meta-analytic approach. Journal of physical activity \& health, 5(3), 385-397. https://doi.org/10.1123/jpah.5.3.385

Macarro, J., Romero, C., \& Torres, J. (2009). Motivos de abandono de la práctica de actividad físico-deportiva en los estudiantes de Bachillerato [Reasons for abandoning the practice of physical-sports activity in high school students]. Revista de Educación, 343, 495-519. http://www.revistaeducacion.educacion.es/re353/re353 18.pdf

Moreno, J. A., \& Martínez, A. (2006). Importancia de la teoría de la autodeterminación en la práctica físico-deportiva: Fundamentos e implicaciones prácticas [Importance of the theory of self-determination in physical-sports practice: Foundations and practical implications]. Cuadernos de Psicología del Deporte, 6(2). Recuperado a partir de https://revistas.um.es/cpd/article/view/113871

Navarro-Aburto, B, Díaz-Bustos, E, Muñoz-Navarro, S, \& Pérez-Jiménez, J. (2017). Condición física y su vinculación con el rendimiento académico en estudiantes de Chile [Physical condition and its relationship with academic achievement in Chilean students]. Revista Latinoamericana de Ciencias Sociales, Niñez y Juventud, 15(1), 309-325. https://doi.org/10.11600/1692715x.1511902032016

Reeve, J. (2016). Motivación y Emoción [Motivation and Emotion]. México: Mc Graw Hill.

Roach, A. (2018). Supportive Peer Relationships and Mental Health in Adolescence: An Integrative Review. Issues in mental health nursing, 39(9), 723-737. https://doi.org/10.1080/01612840.2018.1496498 